\title{
Application of multi-element and multi-isotopic approaches to the study of oceanic anoxia
}

\author{
MingzhaO Sun' COREY ARCheR' DeReK VANCE ' \\ YANAN SHEN ${ }^{2}$
}

Institute of Geochemistry and Petrology, Dept. of Earth

Sciences, ETH Zurich (mingzhao.sun@erdw.ethz.ch)

${ }_{2}$ School of Earth and Space Sciences, University of Science and Technology of China, Hefei, China

Increasingly, multiple proxy or multiple isotope approaches are being used to tackle questions in the Earth Sciences. Furthermore, the stable isotope systems of many of the transition metals have emerged as tracers of many of these processes. A new multi-step chemical separation procedure, validated using multiple rock and other standards, was applied to the study of black shales and carbonates spanning the end-Ordovician, a period of large-scale global change. This method allows us to quantitatively, and with negligible blank contribution, isolate and measure the isotope systems of the transition metals $\mathrm{Fe}, \mathrm{Ni}, \mathrm{Cu}, \mathrm{Zn}$, and $\mathrm{Mo}$ at high precision, from a single sample aliquot and from a range of sample matrix types.

Here, we apply these methods to track the oceanic response to changes in oceanic anoxia across the End-Ordovician mass extinction event. We separate bulk shale sediment samples from Vinini Creek section (Vinini Formation, Nevada, USA), into an HF-digestible fraction, (HFD), and an organic-sulphide-rich fraction (OPF). Separation into distinct phases allows more detailed insights into how these metals are partitioned into different pools, with potential added information to the water column from which these sediments were derived. We use $\mathrm{C}, \mathrm{Zn}, \mathrm{Cu}$ and $\mathrm{Ni}$ elemental and isotopic systematics to explore the links between expanded glaciation, oceanic anoxia and changes to the dynamics of the biological pump.

In this study, preliminary data shows a good linear relationship between the Ni abundance in OPF and TOC in bulk sediments. This relationship is similar to that found in modern upwelling margin reducing sediments' and suggests that the dominant control on $\mathrm{Ni}$ abundances in organic-rich sediments is the supply to the sediment of photosynthesized organic matter. In contrast to $\mathrm{Ni}$, there is no apparent systematic relationship between $\mathrm{Zn}$ abundance and TOC in bulk sediments in the Vinini Creek section, as is also found in modern sediments. This indicates a more complex relationship between $\mathrm{Zn}$ and TOC abundances in reducing sediments. Metal isotope data for the HFD fraction

throughout the Katian $P$. pacificus zone, before the EndOrdovician mass extinction event, are similar to bulk Earth values.

1. Ciscato, E. R., et al. (2018). Earth and Planetary Science Letters 494, 239-250. 
This abstract is too long to be accepted for publication.

Please revise it so that it fits into the column on one page. 